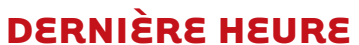

\section{Les cellules souches embryonnaires humaines révèlent l'existence d'une région hautement instable du génome}

Nathalie Lefort, Maxime Feyeux, Cécile Bas, Olivier Féraud, Annelise Bennaceur-Griscelli, Gérard Tachdjian, Marc Peschanski, Anselme L. Perrier

\section{Accumulation d'anomalies} caryotypiques au cours de la culture des cellules $E S$ humaines In vivo, le taux de mutations spontanées dans une cellule somatique normale (contenant environ $3 \times 10^{9}$ nucléotides) est de l'ordre de $10^{-7}$ à $10^{-8}$ par division cellulaire [1] soit environ 100 mutations par cellule à chaque cycle cellulaire. Certaines de ces mutations procurent aux cellules mutées un avantage sélectif qui leur permet de croître plus rapidement. In vitro, lors de la culture à long terme, il en résulte la substitution progressive des cellules non mutées initiales par les cellules porteuses de la mutation. Les cellules souches embryonnaires humaines (ES) sont des cellules pluripotentes dérivées à partir de cellules de la masse interne d'embryons âgés de quelques jours (stade blastocyste) [11, 12]. Elles ont la capacité de se différencier en tous les types cellulaires représentés dans les tissus de l'organisme (pluripotence) et de se multiplier en théorie de manière illimitée in vitro (autorenouvellement). Cette dernière propriété n'a d'intérêt que si l'intégrité du génome des cellules ES peut être maintenue lors des cultures à long terme.

Dès 2004, plusieurs études ont démontré que les conditions de culture classiques des cellules ES humaines in vitro permettant le maintien de leur état indifférencié, induisaient de façon non négligeable une instabilité chromosomique. Des trisomies des chromosomes 12,17 ou $X$ ont ainsi été mises en évidence très fréquemment [2, 3]. L'hypothèse qui prévaut actuellement pour expliquer ce phénomène est que l'amplification de certains gènes portés par ces chromosomes confère un avantage sélectif aux cellules souches indifférenciées. La méthode la plus couramment utilisée pour mettre en évidence ces aberrations chromosomiques est la cytogénétique conventionnelle fondée sur la détection de bandes spécifiques de chaque chromosome (bandes $\mathrm{G}$ ). Le pouvoir résolutif de cette technique ne permet cependant de détecter que les réarrangements chromosomiques de taille supérieure ou égale à 5 à 10 mégabases (Mb).

\section{Les caryotypes moléculaires}

\section{révèlent des aberrations}

chromosomiques plus discrètes

Dans une publication récente parue dans Nature Biotechnology [4], nous décrivons une région génomique hautement instable identifiée grâce à un contrôle régulier de l'intégrité du génome de cellules souches embryonnaires humaines maintenues en culture. Pour cette étude nous avons choisi de contrôler la stabilité chromosomique des cellules à l'aide de deux techniques de «caryotype moléculaire» combinant cytogénétique et biologie moléculaire qui permettent la détection de variations du
N. Lefort, M. Feyeux, M. Peschanski,

A.L. Perrier : Inserm/UEVE UMR-861,

I-STEM, AFM, Institut

des cellules Souches pour le Traitement et l'Étude des maladies Monogéniques,

5 , rue Henri Desbruères,

91030 Évry Cedex, France.

aperrier@istem.genethon.fr

nlefort@istem.genethon.fr

C. Bas, G. Tachdjian : Service de biologie

et génétique de la reproduction,

Hôpital Antoine Béclère, Université

Paris-Sud 11, Clamart, France.

Inserm U935, plate-forme des cellules

souches pluripotentes/

Université Paris-Sud 11,

Hôpital Paul Brousse, Villejuif, France.

0 . Féraud, A. Bennaceur-Griscelli :

Inserm U935, plate-forme

des cellules souches pluripotentes/

Université Paris-Sud 11,

Hôpital Paul Brousse, Villejuif, France.

nombre de copies de fragments d'ADN de tailles inférieures à une mégabase. Ces technologies sont l'hybridation génomique comparative sur puce à ADN (ou microarray $\mathrm{CGH}$ ) et les puces à SNP (single nucleotide polymorphism).

Cinq lignées de cellules $\varepsilon S$ humaines ( $\mathrm{HI}$, H9, SAO1, VUBO1 et VUB05-HD) ont été cultivées pendant plusieurs mois in vitro dans deux laboratoires indépendants et elles ont été régulièrement contrôlées par une technique de caryotype moléculaire (Figure 1). Une copie surnuméraire d'un fragment du chromosome 20 (20q11.21), d'une taille comprise entre 2,5 et $4 \mathrm{Mb}$ selon les lignées, a été détectée dans quatre de ces lignées ( $\mathrm{H1}, \mathrm{H9}, \mathrm{SAOl}$ et VUB05-HD). Celle-ci est sélectionnée au cours des passages successifs des lignées. En utilisant une technique d'hybridation in situ fluorescente (FISH) d'une sonde recouvrant la région amplifiée, nous avons déterminé que la copie surnuméraire du fragment de chromosome 20 était soit répétée - en tandem ou inversée - sur le chromosome 20 (pour les lignées H9, SA01, VUB05-HD) (Figure 2A, B) soit insérée en 1 p36 (pour la lignée $\mathrm{Hl}$ ) (Figure 2C). Ces observations sont corroborées par une étude réalisée par une équipe de l'université libre de Bruxelles publiée en 


\begin{tabular}{|c|c|c|c|c|c|}
\hline \multirow[b]{2}{*}{ Lignée } & \multirow[b]{2}{*}{ Origine } & \multirow[b]{2}{*}{ Caryotype } & \multicolumn{2}{|c|}{$\operatorname{dup}(20)(q 11.21)$} & \\
\hline & & & $\begin{array}{c}\text { Plus haut } \\
\text { passage négatif }\end{array}$ & $\begin{array}{c}\stackrel{+}{\text { Plus bas }} \\
\text { passage positif }\end{array}$ & Références \\
\hline $\mathrm{Hl}$ & WiCell & $46, x y$ & 80 & 24 & {$[4,6]$} \\
\hline HSFl & UCSF & $46, x y$ & - & 29 & {$[7]$} \\
\hline SA01 & Cellartis & $546, x y$ & 41 & 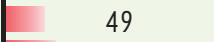 & [4] \\
\hline $\mathrm{H} 7$ & WiCell & $46, x y$ & 41 & 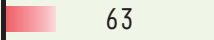 & [6] \\
\hline H9 & WiCell & $46, X X$ & 74 & 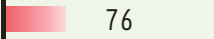 & [4] \\
\hline VUB07 & $A Z-V U B$ & $46, X X$ & 32 & 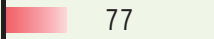 & [5] \\
\hline VUB03-DMI & $A Z-V U B$ & $46, x X$ & 30 & 98 & [5] \\
\hline VUB09-FSHD.1 & I AZ-VUB & $46, X X$ & 28 & 100 & {$[5]$} \\
\hline VUB05-HD & $A Z-V U B$ & $46, x y$ & 106 & 103 & {$[4,5]$} \\
\hline VUBOI & $A Z-V U B$ & $46, x y$ & 126 & 135 & {$[4,5]$} \\
\hline VUB02 & $A Z-V U B$ & $46, x y$ & 219 & 252 & [5] \\
\hline VUB04-CF & $A Z-V U B$ & $46, X X$ & 29 & - & [5] \\
\hline VUB06 & $A Z-V U B$ & $46, x X$ & 64 & - & [5] \\
\hline VUB11-FXS & $A Z-V U B$ & $46, X X$ & 45 & - & [5] \\
\hline VUB13-FXS & $A Z-V U B$ & $46, X X$ & 28 & - & [5] \\
\hline VUB15 & $A Z-V U B$ & $46, x y$ & 18 & - & [5] \\
\hline VUB17 & $A Z-V U B$ & $46, X X$ & 7 & - & [5] \\
\hline VUB19-DMI & $A Z-V U B$ & $46, X X$ & 32 & - & [5] \\
\hline VUB24-DMl & $A Z-V U B$ & $46, X X$ & 31 & - & [5] \\
\hline
\end{tabular}

Médiane des plus hauts passages négatifs connus

37

Figure 1. Détection d'une duplication de la région 20q11.21 dans des cellules souches embryonnaires humaines par différents groupes.

même temps que nos observations [5]. C. Spits et al., décrivent la présence, dans un tiers des lignées ES humaines testées, de la même duplication chromosomique de la région 20q11.21. Cette amplification de la région 20 qll.21 avait déjà été décrite de manière isolée à deux reprises. La première fois dans l'une des premières études ayant utilisé la technique de caryotypes moléculaires sur des cellules ES humaines [6], la seconde fois dans un article visant à répertorier les variations non pathologiques de nombre de copies d'ADN présents dans ces lignées [7]. Dans ce dernier cas I'amplification de la région 20q11.21 avait été interprétée comme un polymorphisme non répertorié dans les bases de données et préexistant à la culture.

\section{La région $20 \mathrm{q} 11.21$}

\section{est fréquemment}

\section{amplifiée dans les cancers}

La région 20q11.21 humaine amplifiée dans les cellules ES humaines en culture à long terme contient une vingtaine de gènes codant pour des protéines et un microARN. la progression tumorale et lors de la culture à long terme des cellules ES humaines. Ces gènes pourraient jouer un rôle dans la prolifération, la survie ou la mort cellulaires. Parmi ces gènes, IDI (inhibitor of DNA binding 1), impliqué dans la croissance des cellules, BCL2LL, impliqué dans la survie et la mort cellulaires, PDRG1 ( $p 53$ and DNA damage regulated 1 ) contrôlé par $p 53$, ou encore POFUTl (protein 0-fucosyltransferase 1) fortement exprimé dans les glioblastomes, représentent autant de candidats pouvant contribuer à l'avantage sélectif de cette instabilité. Le micro ARN hsa-mir1825 localisé dans l'un des exons du gène POFUTl est un autre bon candidat car les microARN sont souvent décrits comme des suppresseurs de tumeurs ou, à l'inverse, des oncogènes [13].

\section{Prolifération des cellules souches:} vers une meilleure compréhension des processus cellulaires liés aux cancers?

Les cellules souches embryonnaires humaines peuvent donc contribuer à une meilleure compréhension des événements précoces jouant un rôle dans la progression tumorale. Elles présentent l'avantage de permettre d'étudier l'implication de la région identifiée dans un contexte dans lequel il n'existe pas d'autres anomalies génétiques ce qui est rarement le cas lorsque des cellules de biopsies de tumeurs humaines sont étudiées. Dans le cadre de l'application thérapeutique directe de cellules ES humaines ou de leur descendance (thérapie cellulaire) comme dans celui de l'utilisation de ces cellules pour la recherche, nos résultats sur l'instabilité génétique relative des cellules $\varepsilon S$ humaines encouragent à intégrer le caryotypage moléculaire dans le contrôle qualité. $\diamond$ A recurrent hotspot of genomic instability identified in human ES cells

\section{REMERCIEMENTS}

Ce travail a été soutenu par: MediCen (programme Inge(ell), Université Paris Sud 11, FP6 de l'EC (STEM-HD) et ANR (hESCREEN). VUBOI et VUB05-HD ont été produites par le Dr Karen Sermon de l'AZ-VUB (Bruxelles). 

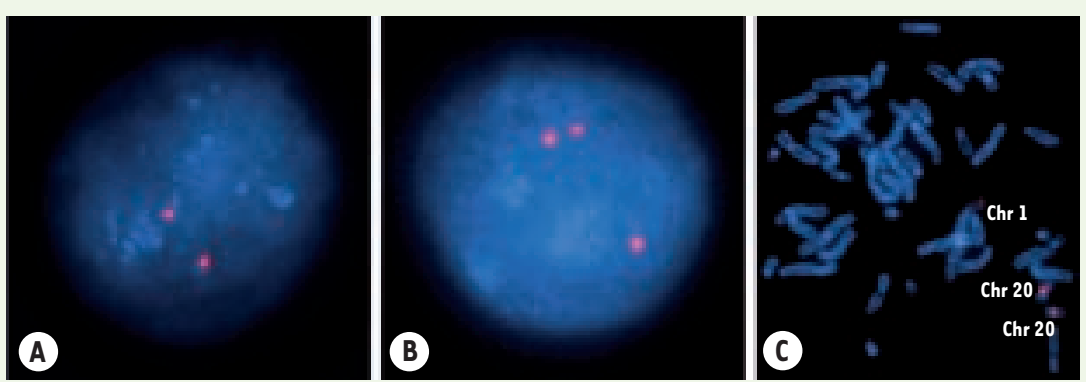

Figure 2. Analyse par FISH de l'instabilité génétique en 20q11.21. A. Deux signaux détectés sur noyaux interphasiques à l'aide de sondes spécifiques de la région 20q11.21. B. Trois signaux détectés sur noyaux interphasiques correspondant à la duplication de la région $20 q 11.21$ du chromosome 20. C. Ins $(1 ; 20)$ (p36.3;q11.21) : trois signaux détectés sur chromosomes métaphasiques mettant en évidence une insertion de la région 20q11.21 dans le bras court du chromosome l (1p36.3).

\section{RÉFÉRENCES}

1. Kondrashov AS. Direct estimates of human per nucleotide mutation rates at 20 loci causing Mendelian diseases. Hum Mutat 21 (2003) 12-27.

2. Baker DE, Harrison NJ, Maltby $\varepsilon$, et al. Adaptation to culture of human embryonic stem cells and oncogenesis in vivo. Nat Biotechnol 2007; $25: 207-15$

3. Draper JS, Smith K, Gokhale P, et al. Recurrent gain of chromosomes $17 \mathrm{q}$ and 12 in cultured human embryonic stem cells. Nat Biotechnol 2004 ; 22 : 2253-4.
4. Lefort N, Feyeux M, Bas C, et al. Human embryonic stem cells reveal recurrent genomic instability at 20q11.21. Nat Biotechnol 2008; 26 : 1364-6.

5. Spits C, Mateizel I, Geens M, et al. Recurrent chromosomal abnormalities in human embryonic stem cells. Nat Biotechnol 2008; $26: 1364-6$.

6. Maitra A, Arking DE, Shivapurkar N, et al. Genomic alterations in cultured human embryonic stem cells. Nat Genet 2005 ; 37 ; 1099-103.

7. Wu H, Kim KJ, Mehta K, et al. Copy number variant analysis of human embryonic stem cells. Stem Cells $2008 ; 26: 1484-9$.
8. Rithidech K, Bond VP, Cronkite EP, et al.

Hypermutability of mouse chromosome 2 during the development of $x$-ray-induced murine myeloid leukemia. Proc Natl Acad Sci USA 1995 ; 92 : 1152-6.

9. Tanner MM, Tirkkonen M, Kallioniemi A, et al. Independent amplification and frequent co-amplification of three nonsyntenic regions on the long arm of chromosome 20 in human breast cancer. Cancer Res 1996; $56: 3441-5$.

10. Scotto L, Narayan G, Nandula SV, et al. Identification of copy number gain and overexpressed genes on chromosome arm $20 \mathrm{q}$ by an integrative genomic approach in cervical cancer: potential role in progression. Genes Chrom Cancer $2008 ; 47: 755-65$.

11. Fluckiger AC, Dehay C, Savatier P. Cellules souches embryonnaires et thérapies cellulaires du système nerveux. Med Sci (Paris) 2003 ; $19: 699-708$.

12. Coulombel L, De Vos J, Pucéat M. FISH-ESC: Premier colloque international sur la recherche dédiée aux cellules souches embryonnaires humaines. Med Sci (Paris) $2008 ; 24: 419-26$.

13. Finoux AL, Chartrand P. Micro-ARN : oncogènes et suppresseurs de tumeurs. Med Sci (Paris) 2008 $24: 1049-54$

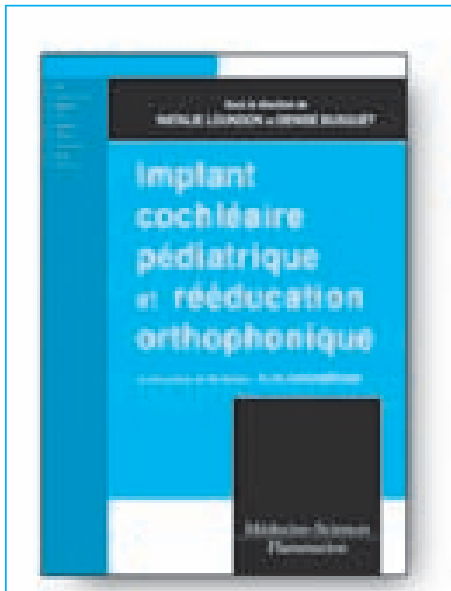

28 jancier 2009

144 payes -25 illuatudien Pit potle rfe: $35 \mathrm{C}$

\section{Implant cochléaire pédiatrique et rééducation orthophonique}

Natalie LOUNDON, midecin ORL ay service gropl ef de chinurge cervicotaciak de fndpital gentants Amand Trosseau

Cet ouvrage de grande actualité rópood aux questions des équipes en charge des enfants sounds implantós, tan sur le plan intelical, ithilusatif et fanillial que woluirs.

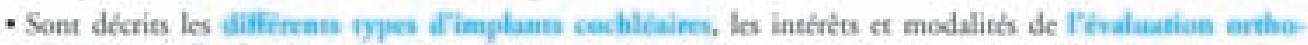
phumique de l'enfant apeós la pose de l'implant.

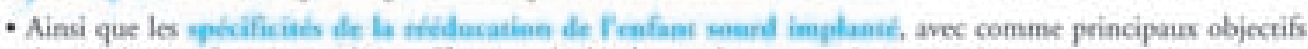
de reteablir une foncrion audirive efficace er de developper la coenmunication oeale.

- Acruel et accessible, louvrage expose en outre de nombreas cas dinhanes, sous forme de fiehes juratiques er de mises eat simustion.

\section{Au total, un livre original, unique en langue française et indispensable à la prise en charge de l'enfant sourd implanté cochléaire.}

En vente chez votre fibraire specialise, par correspondance ou sur notre site www.medecine, flammarion.com

K Bon de commande à retourner completè a : FLAMMARION Medecine-Sciences - 87, quai Panhard et Levassor - 75647 Paris cedex 13

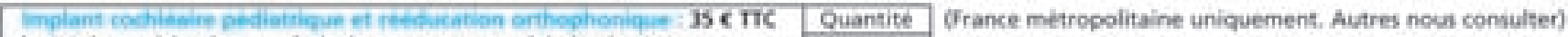
( +5 C de participatien aux trais de port par exemplaire) soit $40 \mathrm{C}$ le jeirs mon reglement a la commande: Montant total de :

Cheque bancaire ou pontal payable en France a l'orde de Flammarion SA (Une facture acquitte vera jointe au colis)

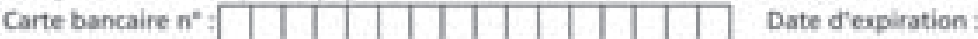

Les 3 demien chiftres situts au dos de votre carte bancaire:

Nom $f$ Prènom

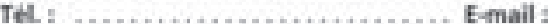

Date et vignature obligatoire:

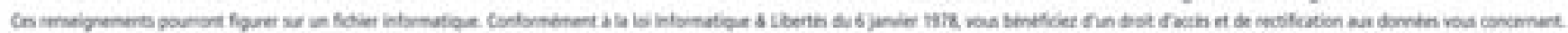

